\title{
Graphene: a fascinating material
}

\author{
S. Sahoo ${ }^{1}$ and S. K. Sahoo ${ }^{2}$ \\ ${ }^{1}$ Department of Physics, National Institute of Technology, Durgapur - 713209, West Bengal, India. \\ ${ }^{2}$ Department of Physics, Kalinga Institute of Industrial Technology University, Bhubaneswar - 751024, Orissa, India \\ sukadevsahoo@yahoo.com, physushant@gmail.com
}

Abstract: Graphene is the first two-dimensional allotrope of carbon. Recent theoretical studies of graphene reveal that the linear electronic band dispersion near the Brillouin zone corners give rise to electrons and holes that propagate as if they are massless fermions and anomalous quantum transport is observed experimentally. Graphene has potential for serving as an excellent electronic material that can be used in place of silicon for making ultrafast and stable transistors. It is considered as a promising candidate for electronics and spintronics applications. It provides a bridge between condensed matter physics and quantum electrodynamics.

Keywords: Carbon, graphene, Dirac fermions, anomalous quantum Hall effect.

\section{Introduction}

Carbon is one of the most intriguing elements in the Periodic Table. It has different allotropic forms. Graphene is the recently discovered two-dimensional allotropic from of carbon. Before graphene three-dimensional (diamond, graphite), one-dimensional (nanotubes) and zerodimensional (fullerenes) (Fig. 1) (Katsnelson, 2007) allotropes of carbon were known. Two carbon allotropes diamond and graphite - have been known to humans since ancient times. Fullerenes (Kroto et al., 1985) were discovered in1985, carbon nanotubes (lijima, 1991) in 1991 and graphene (Novoselov et al., 2004a,b) in 2004. Carbon exhibits some remarkable paradoxical properties (Basu, 2008). For example, diamond is the hardest naturally occurring substances known, while graphite is one of the softest substance known. Diamond behaves like an insulator while graphite is a very good conductor of electricity. Both are three-dimensional forms of carbon.

Graphene is a monolayer of carbon atoms packed into a dense honeycomb crystal structure. Graphene sheets are one-atom thick, 2D layers of $s p^{2}$-bonded carbon. In graphene, carbon atoms are arranged in planar and hexagonal form. Graphene has two atoms per unit cell. The carbon-carbon bond length in graphene is $\sim 1.42 A^{0}, 1 A^{0}$ (angstrom) $=0.1$ nanometer or $1 \times 10^{-10}$ meters. Graphene is the building block for carbon materials of all other dimensions (Geim \& Novoselov, 2007) therefore it is known as the mother of all graphitic materials (Srinivasan, 2007). Graphite is obtained by the stacking of graphene layers. Diamond can be obtained from graphene under extreme pressure and temperatures by transforming the 2-dimensional $\mathrm{sp}^{2}$ bonds into 3dimensional $\mathrm{sp}^{3}$ bonds. Carbon nanotubes are synthesized from rolled up graphene. Fullerenes can also be obtained from graphene by modifying the hexagons into pentagons and heptagons in a systematic way.

In 2004, a group of physicists of Manchester University, UK, led by Andre Geim and Kostya Novoselov (Novoselov et al., 2004a,b), used three-dimensional graphite for their experiment and extracted a single sheet (a monolayer of atoms) of graphite, which is known as graphene. To obtain graphene they used a technique known as 'micromechanical cleavage'. Perfect graphene is in hexagonal form but imperfections can cause heptagonal or pentagonal structures.

The honey comb lattice of graphene consists of two interpenetrating triangular sublattices (Fig. 2a) (Geim \& MacDonald, 2007). The sites of one sublattice (green) are at the centers of triangles defined by the other (orange). Thus the lattice has two carbon atoms ( $A$ and $B$ ) per unit cell and is invariant under $120^{\circ}$ rotations around any lattice site. Each atom has one $s$ and three $p$ orbitals. The $s$ orbital and two in-plane $p$ orbitals are tied up in graphene's strong covalent bonding and do not contribute to its conductivity. The remaining $p$ orbital, perpendicular to the molecular plane, is odd under inversion in the plane and hybridizes to form $\pi$ (valence) and $\pi^{\prime}$ (conduction) bands (Fig. 2b). These two $\pi$ bands are needed to describe the electronic properties of graphene. The $\pi$ and $\pi^{\prime}$ bands (blue in the electronic structure) are decoupled from the $\sigma$ and $\sigma^{\prime}$ bands (red) because of inversion symmetry and closer to the Fermi energy. The Fermi energy separates occupied and empty states. In a neutral graphene sheet, this is equal to zero energy since valence and conduction bands meet (known as neutrality point). The bands form conical valleys that touch at two of the high symmetry points (usually labelled by their momentum vector $K$ and $K^{\prime}$ ) in the Brillouin zone, which makes graphene a gapless semiconductor. Near these points the energy varies linearly with the magnitude of momentum measured from the Brillouin zone corners. The four other Brillouin zone corners are related to $K$ and $K^{\prime}$ by reciprocal lattice vectors and do not represent distinct electronic states.

Electrons in graphene are obeying a linear dispersion relation (i.e. the electron energy is linearly proportional to the wave vector, $E=\hbar k v_{F}$ ) and behave as massless relativistic particles, called Dirac fermions. $\hbar=h / 2 \pi, h$ is the Planck's constant, $\mathrm{k}$ is the wave vector and $v_{F}$ is the Fermi velocity of electron in the graphene. This property implies that the speed of electrons in graphene 
is a constant, independent of momentum, like the speed of photons is a constant $c$. Recently it is found that the velocity of electrons in graphene is about $10^{6} \mathrm{~m} \mathrm{~s}^{-1}$. This velocity is large but still 300 times slower than the velocity of light in vacuum $c$. Because the electrons are sluggish compared to the speedy photons they exchange when interacting, the physics of electron-electron interaction in graphene is different from that of photon-mediated interactions between fermions in quantum electrodynamics (QED). In graphene the interactions among electrons are extremely strong and graphene's dimensionless coupling constant $\alpha_{G R}=e^{2} / \hbar v_{F} \approx 1$ is larger than the dimensionless coupling constant of QED, $\alpha=e^{2} / \hbar c \approx 1 / 137$. The large difference between $\mathrm{c}$ and $v_{F}$ implies that the interacting electrons in a graphene sheet is not like the 2D version of QED, for example not Lorentz invariant. Again the interaction of electrons in graphene is also different from an ordinary nonrelativistic $2 \mathrm{D}$ electron gas. The electrons in most of the conductors can be described by non-relativistic quantum mechanics but the electrons in graphene are treated as relativistic particles and are described by the Dirac equation rather than Schroedinger equation. This shows a possibility of studying phenomena of quantum field theory in condensed matter physics. Graphene's relativistic behaviour arises not from special relativity but simply from the symmetry of the honeycomb lattice. All this makes graphene a new type of electronic system whose independent particles move relativistically, but interact nonrelativistically (Geim \& MacDonald, 2007).

\section{Properties of graphene}

Here, we will discuss only some peculiar properties of graphene. Graphene has high mobility at room temperature, with values of $15000 \mathrm{~cm}^{2} V^{-1} s^{-1}$. These high mobilities are independent of temperature between 10 and $100 K$. Recent results suggest that mobilities greater than $200,000 \mathrm{~cm}^{2} V^{-1} s^{-1}$ are possible if extrinsic disorder can be eliminated. Recent experimental data suggests that doping does not influence the carrier mobility in graphene. Even for chemical dopant concentrations in excess of $10^{12} \mathrm{~cm}^{-2}$ there is no observable change in the carrier mobility.

It is shown that graphene has a minimum electrical conductivity of the order of the quantum unit $e^{2} / h$, even when the concentration of charge carriers is zero (Novoselov et al., 2005; Zhang et al., 2005), where $e$ is the charge of the electron. The electrical conductivity of graphene does not fall below this minimum value. The origin of this minimum conductivity is still unclear; however, rippling of the graphene sheet may lead to local puddles of carriers that allow conduction. Strong interactions among electrons near the neutrality point might play a role. This is completely counterintuitive because in all other systems the conductivity is zero if no charge carriers are present.

Graphene shows very interesting behavior in the presence of a magnetic field at very low temperature, typically below $-243^{\circ} \mathrm{C}$ (Basu, 2008). Graphene shows an anomalous quantum Hall effect with the sequence shifted by $1 / 2$ with respect to the standard sequence. Here, we want to write a few sentences about the quantum Hall effect (QHE). The quantum Hall effect is one the most remarkable phenomena in condensed matter physics discovered in the second half of the $20^{\text {th }}$ century. The basic fact characterizing quantum Hall effect is that the diagonal electric conductivity of a twodimensional electron system in a strong magnetic field is vanishingly small $\sigma_{x x} \rightarrow 0$, while the non-diagonal conductivity is quantized in multiples of $e^{2} / h$ : $\sigma_{x y}=p e^{2} / h$ (Gusynin \& Sharapov, 2005), where $p$ is an integer (the integral quantum Hall effect, IQHE). When $p$ is a fractional number, it is known as fractional quantum Hall effect (FQHE). One of the author has discussed both IQHE and FQHE in 2D electron gas briefly in another popular article (Sahoo \& Goswami, 2007). In recent experiments (Zhang et al., 2005), the quantum Hall effect is observed in graphene. It is found that the Hall conductivity $\sigma_{x y}= \pm 4 e^{2} / h\left(N+\frac{1}{2}\right)$

(Geim \& MacDonald, 2007; Novoselov et al., 2005; Zhang et al., 2005), where $N$ is the Landau level index and the factor 4 accounts for graphene's double spin and double

Fig. 1. Crystal structures of the different allotropes of carbon. (Left to right) Three-dimensional diamond and graphite (3D);

two-dimensional graphene (2D); one dimensional nanotubes (1D); and zero-dimensional buckyballs (OD) (Katsnelson, 2007).

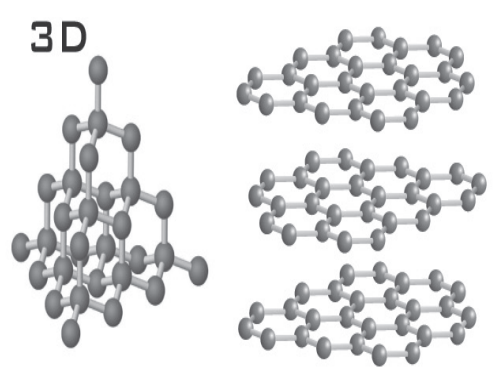

Popular article

COIndian Society for Education and Environment (iSee)
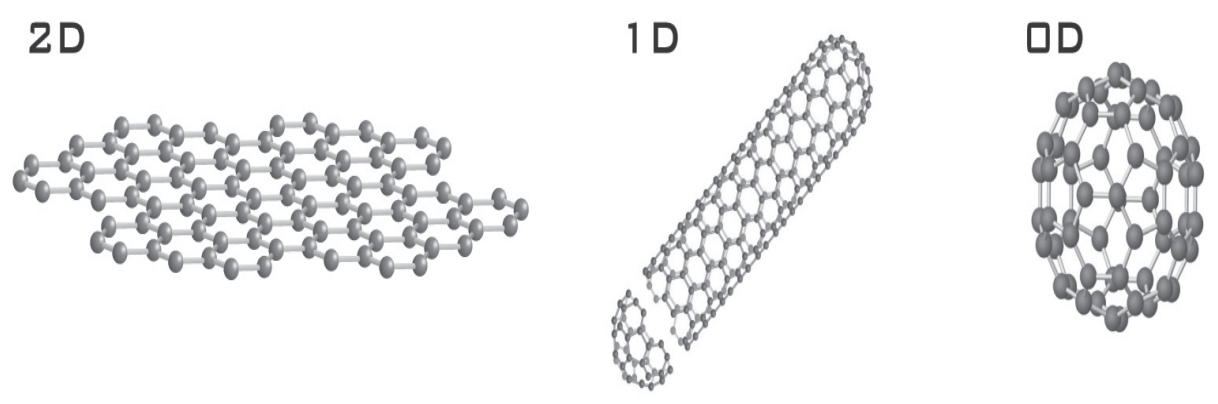

Sahoo \& Sahoo Indian J.Sci.Technol. 
band (valley) degeneracy. That is why; it is characterized as half-integer quantum Hall effect. The first plateau occurs at $2 e^{2} / h$. The half-integer QHE in graphene has also been suggested by theory groups (Gusynin \& Sharapov, 2005; Peres et al., 2006). This anomalous
Chakraborty, 2006; Toke \& Jain, 2007; Goerbig \& Regnault, 2007; Yang, 2007; Papic et al., 2009; Sahoo \& Das, 2009a). Possible fractional quantum Hall states in graphene have been discussed there. The possible supersymmetric structure of fractional quantum Hall effect

Fig. 2. Crystal and electronic structures of graphene (Geim \& MacDonald, 2007)

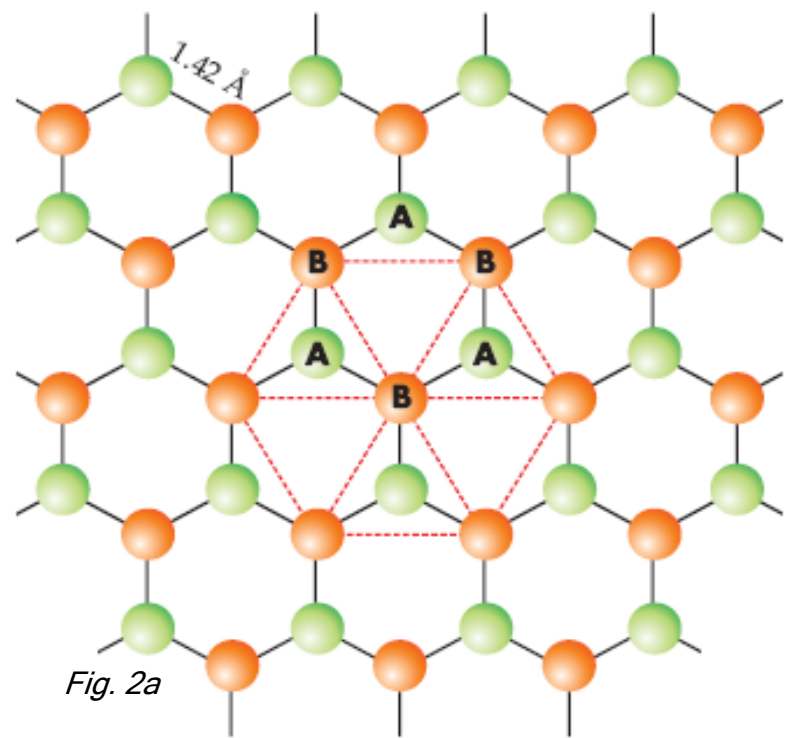

QHE is the direct evidence for Dirac fermions in graphene. The QHE in bilayer graphene is more interesting. Interestingly, the first plateau at $N=0$ is absent and the first plateau appears at $4 e^{2} / h$, as in the conventional QHE. In general, the quantized plateaus appear at the standard sequence $\sigma_{x y}= \pm 4 N e^{2} / h$ (same as the nonrelativistic electrons) with a missing plateau at zero energy (Fig. 3) (Geim \& MacDonald, 2007), so that the step in the Hall conductance separating electron- and hole- like regions is twice as large as the quantized steps on either side of the charge neutral state. This anomaly at $E=0$ can be removed by field effect doping, which has the effect of adding carriers and splitting the layer degeneracy of the zero energy Landau level producing two new steps in the Hall conductance, each of height $4 e^{2} / h$. This unusual quantization in bilayer graphene leads new elementary excitations called massive Dirac fermions. These fermions have quadratic dispersion, like massive nonrelativistic particles, and described by an off diagonal, Dirac like Hamiltonian (Geim \& Novoselov, 2007; Geim \& MacDonald, 2007). Further, it is also found that room-temperature QHE (Novoselov et al., 2007) can be observed in graphene. The observation of QHE in graphene at room temperature opens up new vistas for graphene based resistance standards and quantum devices. Although the FQHE has not yet been observed in graphene, it has been explored theoretically in a number of papers (Peres et al., 2006; Toke et al., 2006; Yang et al., 2006; Apalkov \&

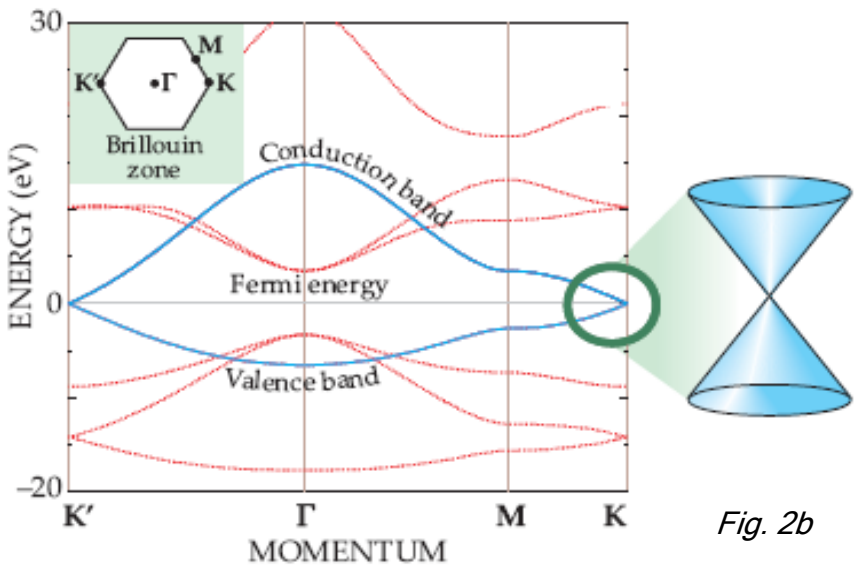

in graphene is discussed by one of the authors (Sahoo \& Das, 2009b) which may be checked experimentally in future. It is also found that superconductivity can be induced in graphene (Heersche et al. 2006, 2007).

Graphene sheets are held together by van der Waals forces. It is found that graphene has a spring constant of the order of $1-5 \mathrm{~N} / \mathrm{m}$ and its Young's modulus is $0.5 \mathrm{TPa}$. Due to these high values, graphene is very strong and rigid. Graphene is considered as the strongest material ever tested till today. Due to these intrinsic properties graphene is used as pressure sensors, and resonators etc. Now we consider about thermal conductivity. Thermal conductivity of graphene is found to be between $(4.84 \pm 0.44) \times 10^{3}-(5.30 \pm 0.48) \times 10^{3} \mathrm{Wm}^{-1} \mathrm{~K}^{-1}$ at room temperature. This value is a factor of $\sim 100$ larger than the thermal conductivity of graphite. Thus, graphene has high mechanical and thermal properties.

Graphene is an ideal material for spintronics due to small spin-orbit interaction and absence of nuclear magnetic moments in carbon. Nanostripes of graphene at low temperature show spin-polarized edge currents which are used in the field of spintronics.

\section{Applications}

Graphene has a large number of applications. A few of them are discussed below:

Transistors: Graphene can be used to make excellent transistors. It is so thin we can easily control whether or not it conducts by applying an electric field. Electrons in graphene also travel ballistically over sub-micron 
distances. As a result, graphene-based transistors can run at higher frequencies and more efficiently than the silicon transistors we use now. At the present moment we have no way to produce entire integrated circuits from these transistors since we are limited by the size of graphenes we can produce. Graphene can be used to create world's smallest transistor. The smallest transistor so far, 1 atom thick and 10 atoms wide, was made in 2008 (Ponomarenko et al., 2008; Wikipedia, 2009).

Gas Sensors: Gas molecules that land on graphene affect its electronic properties in a measurable way - in fact, we can measure the effect of a single molecule associating with a graphene. This means that we can create gas sensors which are sensitive to a single atom or molecule.

Support membranes for transmission electron microscopy. Graphene is effectively the thinnest material (Jacoby \& Chicago, 2009) that we can make out of atoms. Suprisingly it is also very strong. Carbon nanotubes are made from rolled up graphene, and it has been suggested that cabling made from nanotubes would be strong enough to create an elevator into space!. As a result we can use it to hold micro- and nanoscopic objects we wish to look at in an electron microscope (e.g. DNA, nanoparticles) in a similar way we use glass slides in an optical microscope. Graphene is the perfect material for this job as it is made only of carbon, it is very thin so will not interfere with the pictures taken as much as other materials, and has a very simple crystal structure so can easily be eliminated from diffraction patterns.

Inert coatings: Graphene is resistant to attack by many powerful acids and alkalis such as hydrofluoric acid and ammonia. So it could be used to give objects an atomically thin protective coating which would provide protection against these agents.

Molecular sieves. The open honeycomb structure of graphene means it might be possible to use it as a net or sieve for atoms and small molecules, since only objects of this size will be able to fit through the lattice. It could therefore be used in a way analogous to a filter paper, trapping large molecules and allowing smaller ones to pass.

Graphene nanoribbons: Graphene nanoribbons (GNRs) (Wikipedia, 2009) are single layers of graphene that are cut in a particular pattern to give it certain electrical properties. Due to their 2D structure, high electrical conductivity and thermal conductivity, and low noise GNRs can be used in place of copper for integrated circuit interconnects. Recently it is also found that quantum dots can be created by changing the width of GNRs at select points along the ribbon.

Transparent conducting electrodes: Due to its high electrical conductivity and high optical transparency graphene can be used as transparent conducting electrodes in liquid crystal displays, touchscreens, organic photovoltaic cells, and organic light emitting diodes etc.
Ultracapacitors: Due to the high surface area to mass ratio of graphene, it is used to produce ultracapacitors with greater energy storage density.

Fig. 3. Quantum Hall effect in graphene as a function of charge-carrier density n. (Geim \& MacDonald, 2007)

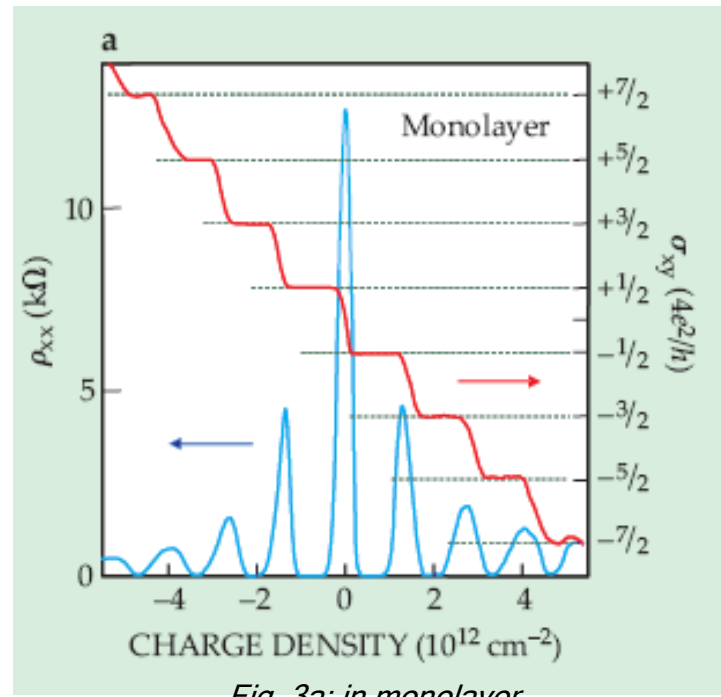

Fig. 3a: in monolayer

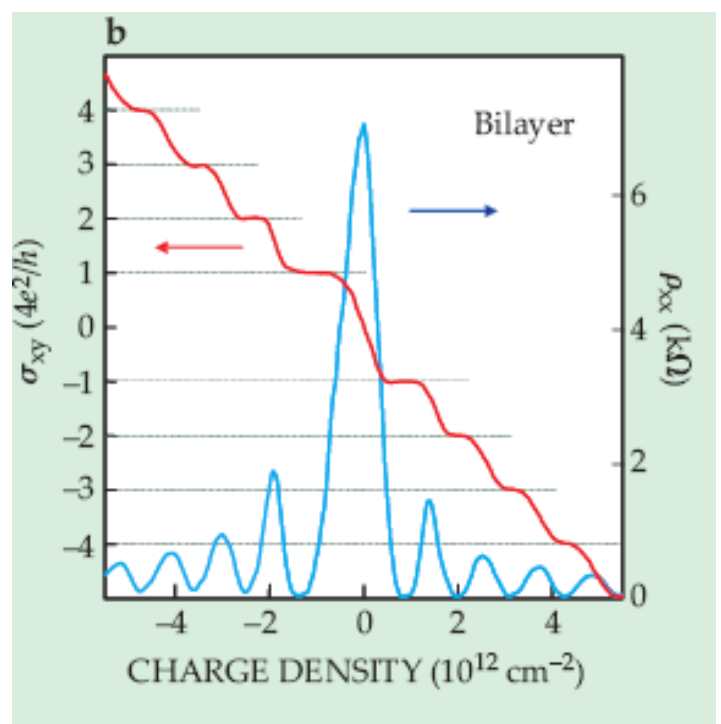

Fig. 3b: in bilayer

\section{Conclusions}

Graphene is one of the fastest growing topics in material science these days. It is the first example of truly atomic 2D crystalline matter. It has some special characteristics: it is a gapless semiconductor with a linear energy spectrum, minimum conductivity even if in the absence of charge carriers, half-integer quantum Hall effect etc. It is also found that superconductivity can be induced in graphene. Graphene is presently one of the 
most expensive materials on Earth. The cost of graphene with a sample smaller than the thickness of a human hair is more than $\$ 1,000$ (as in April 2008). Recently, Marcus Freitag of IBM's T. J. Watson Research Center in Yorktown Heights, New York has said that "graphene is a fascinating material" (Brumfiel, 2009). The study of graphene is a very hot and challenging topic both theoretically and experimentally in condensed matter physics as well as quantum field theory.

\section{References}

1. Apalkov VM and Chakraborty T (2006) Fractional quantum Hall states of Dirac electrons in graphene. Phys. Rev. Lett. 97, 126801-1 - 126801-4.

2. Basu B (2008) Graphene: carbon in two dimensions. Sci. Reporter. 45 (7), 33-35.

3. Brumfiel G (2009) Graphene gets ready for the big time. Nature. 458, 390-391.

4. Geim AK and MacDonald AH (2007) Graphene: exploring carbon flatland. Physics Today. 60, 35-41.

5. Geim AK and Novoselov KS (2007) The rise of graphene. Nature Mater. 6, 183-191.

6. Goerbig MO and Regnault N (2007) Analysis of a SU(4) generalization of Halperin's wave functions as an approach towards a SU(4) fractional quantum Hall effect in graphene sheets. Phys. Rev. B. 75, 2414051 - 241405-4 [arXiv: cond-mat/ 0701661].

7. Gusynin VP and Sharapov SG (2005) Unconventional integer quantum Hall effect in graphene. Phys. Rev. Lett. 95, 146801-1 - 146801-4 [arXiv: cond-mat/0506575].

8. Heersche HB et al. (2006) Presented at Nanophysics: from Fundamentals to Applications, Hanoi, Vietnam (August 2006).

9. Heersche HB et al. (2007) Induced superconductivity in graphene. Solid State Commun. 143, 72-76.

10. lijima $S$ (1991) Helical microtubules of graphitic carbon. Nature. 354, 56-58.

11. Jacoby $M$ and Chicago C \& EN (2009) Graphene: Carbon as thin as can be. www.cen-online.org, pp: 14- 20 (March 2009)

12. Katsnelson Ml (2007) Graphene: carbon in two dimensions. Materialstoday. 10(1-2), 20-27.

13. Kroto HW, Heath JR, O'Brein SC, Curl RF and Smalley RE (1985) $\mathrm{C}_{60}$ : Buckminster fullerene. Nature. 318, 162-163.

14. Novoselov KS et al. (2007) Room-temperature quantum Hall effect in graphene. Science. 315, 1379.

15. Novoselov KS, Geim AK, Morozov SV, Dubonos SV, Zhang Y, and Jiang D (2004b) Room-temperature electric field effect and carrier type inversion in graphene films. arXiv:cond-mat/0410631.

16. Novoselov KS, Geim AK, Morozov SV, Jiang D, Katsnelson MI, Grigorieva IV, Dubonos SV and Firsov AA (2005) Two-dimensional gas of massless Dirac fermions in graphene. Nature. 438, 197-200.
17. Novoselov KS, Geim AK, Morozov SV, Jiang D, Zhang Y, Dubonos SV, Grigorieva IV and Firsov AA (2004a) Electric field effect in atomically thin carbon films. Science. 306, 666-669.

18. Papic Z, Goerbig MO and Regnault N (2009) Theoretical expectations for a fractional quantum Hall effect in graphene. arXiv:0902.3233 [Cond-mat.meshall] 18 Feb 2009.

19. Peres NMR, Guinea F and Castro Neto AH (2006) Electronic properties of disordered two-dimensional carbon. Phys. Rev. B. 73, 125411-1 - 125411-23 [arXiv: cond-mat/0506709].

20. Ponomarenko LA et al. (2008) Chaotic Dirac billiard in graphene quantum dots. Science. 320, 356-358.

21. Sahoo $S$ and Das $S$ (2009a) Fractional quantum Hall effect in graphene. Indian J. Pure \& Appl. Phys. 47 (9), 658-662.

22. Sahoo S and Das S (2009b) Supersymmetric structure of fractional quantum Hall effect in graphene. Indian J. Pure \& Appl. Phys. 47 (3), 186191.

23. Sahoo $S$ and Goswami M (2007) Fractional quantum Hall effect. IAPT Bulletin. 24 (12), 388-391.

24. Srinivasan $C$ (2007) Graphene: mother of all graphitic materials. Curr. Sci. 92, 1338-1339.

25. Töke C and Jain JK (2007) SU(4) composite fermions in graphene: new fractional quantum Hall states. arXiv: cond-mat/0701026.

26. Tőke C, Lammert PE, Crespi VH and Jain JK (2006) Fractional quantum Hall effect in graphene. Phys. Rev. B 74, 235417-1 - 235417-5.

27. Wikipedia-Graphene $\left(12^{\text {th }}\right.$ May 2009$)$.

28. Yang K (2007) Spontaneous symmetry breaking and quantum Hall effect in graphene. Solid State Commun. 143, 27-32 [arXiv:cond-mat/0703757].

29. Yang K, Das Sarma S and MacDonald AH (2006) Collective modes skyrmion excitations in graphene SU(4) quantum Hall ferromagnets. Phys. Rev. B. 74, 075423-1 - 075423-8 [arXiv: cond-mat/0605666].

30. Zhang Y, Tan YW, Stormer HL, and Kim P (2005) Experimental observation of the quantum Hall effect and Berry's phase in graphene. Nature. 438, 201204.
Popular article

CIndian Society for Education and Environment (iSee)
"Graphene"

http://www.indjst.org
Sahoo \& Sahoo

Indian J.Sci.Technol. 